\title{
Conductividad hidráulica en un suelo aluvial en respuesta al porcentaje de sodio intercambiable
}

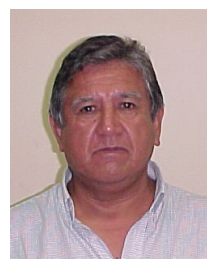

Francisco L. Barreto Filho ${ }^{1}$, Hugo O. Carvallo Guerra² \& Hans R. Gheyi

\author{
1 João Pessoa, Paraíba. Fone: (83) 225-5088. E-mail: linsba@terra.com.br \\ 2 DEAg/CCT/UFCG. CEP 58109-090, Campina Grande, PB. Fone: (83) 310-1185. E-mail: hugo@deag.ufcg.edu.br (Foto)e \\ hans@deag.ufcg.edu.br
}

Protocolo 57 - 26/4/2002 - Aprovado em 14/8/2003

\begin{abstract}
Resumen: El efecto del porcentaje de sodio intercambiable (PSI) sobre la conductividad hidráulica de un suelo saturado, fue estudiado en condiciones de laboratorio a través de la determinación de las relaciones entre la conductividad hidráulica medida en un suelo normal y las medidas en suelos con diferentes PSI. Los resultados muestran una gran reducción de la conductividad hidráulica con el aumento de sodio en el suelo, llegando esta reducción a ser en las muestras más sodificadas de casi $100 \%$, cuando comparadas con las muestras sin sodio, hecho probablemente acontecido debido al efecto dispersante del sodio sobre las partículas del suelo.
\end{abstract}

Palabras clave: conductividad hidráulica relativa, sodio intercambiable, dispersión

\section{Saturated hydraulic conductivity of an alluvial soil with different exchangeable sodium percentages}

\begin{abstract}
The effect of different exchangeable sodium percentages (ESP) on the saturated hydraulic conductivity of a soil was studied under laboratory conditions by determining the relationship between the hydraulic conductivity of a normal soil and that measured on soil with different ESP. The results show a great reduction in the saturated hydraulic conductivity with the increase of the exchangeable sodium percentage in the soil, this reduction being as great as $100 \%$ on the highly sodified samples when compared with those which did not receive sodium treatment. This fact is explained due to the dispersing effect of the exchangeable sodium on the soil particles.
\end{abstract}

Key words: relative hydraulic conductivity, exchangeable sodium, dispersion

\section{INTRODUCCION}

Suelos con problemas de sales ocurren en regiones áridas y semiáridas del noreste del Brasil donde condiciones de baja precipitación y altas temperaturas favorecen su formación. Asociados a estas características climáticas, otros factores tales como baja permeabilidad del suelo, manejo inadecuado de este y agua de riego de baja calidad, contribuyen para aumentar el problema, perjudicando la producción y el desarrollo socioeconómico de la región.

El efecto negativo del porcentaje de sodio intercambiable (PSI) sobre las propiedades físicas del suelo depende de varios factores entre lo cuales la presencia de sales en la solución del suelo, la textura, el tipo de arcilla y la conductividad eléctrica del agua de riego (Sumner, 1995).

Los efectos de la sodicidad en las propiedades de los suelos pueden afectar adversamente el balance ecológico de un área. Estudios han demostrado que un alto PSI en el complejo de cambio del suelo puede provocar dispersión y expansión de las partículas de éste. La dispersión y el consecuente reordenamiento de las partículas del suelo obstruirían los espacios porosos, disminuyendo así la conductividad hidráulica. La expansión de las partículas de arcilla produciría un afecto similar (Frenkel et al., 1978, Rhoades, 1990, Chhabra, 1996). El proceso de dispersión de las arcillas puede ir precedido por la expansión de las mismas, cuando el agua entra en contacto con la superficie de las micelas. Rengasamy \& Sumner (1997) han dado una excelente descripción de cómo las partículas de arcilla sufren expansión, desintegración y dispersión en un medio acuoso y como cada un de estos fenómenos afectan los procesos de compactación y cementación de los suelos. Bar-On et al. (1970) encontró que la adición de una pequeña cantidad de sodio intercambiable a una arcilla saturada con calcio aumenta la movilidad electroforética de la arcilla, sin embargo no aumenta el tamaño de los cristales. A medida que el sodio intercambiable aumenta, el sodio penetra entre las placas de arcilla y desintegra sus estructuras cristalinas. Segundo Shainberg (1990) valores de 
sodio intercambiable de hasta $15 \%$ afectan la expansión de las arcillas. Un aumento encima de $15 \%$ causa la desintegración de las arcillas, aunque Pizarro (1978) y Northcote \& Skene (1972) consideran ese valor como 7\%. McNeal \& Coleman (1966) indican que la conductividad hidráulica podría ser adecuada bajo altos valores de PSI siempre que la concentración de sales del agua de riego se mantenga encima de un determinado valor, sobre el cual las partículas flocularían. Pupisky \& Shainberg (1979) indican que bajo estas condiciones aún habría una disminución de la conductividad hidráulica, provocada ahora por la expansión de las arcillas. Excesivo sodio intercambiable en el suelo asociado con pHs mayores de 8,4 afectan las condiciones físicas de los suelos, perjudicando el movimiento del agua y del aire y consecuentemente el crecimiento de las plantas (Gupta \& Abrol, 1990).

Al definir medidas de prevención y recuperación de suelos afectados por sales es de primordial importancia conocer la relación existente entre el contenido de sales y sodio con aquellos parámetros que determinan la eficiencia del drenaje. Entre estos parámetros, la conductividad hidráulica es talvez el más importante, debido a que esta determina la facilidad con que el agua se mueve en el suelo, fundamental para el crecimiento y desarrollo de las plantas, como también para la recuperación de los suelos sódicos. Por otro lado, el conocimiento cuantitativo de la relación entre la conductividad hidráulica y el porcentaje de sodio intercambiable del suelo puede permitir la introducción de una expresión relacionando el movimiento del agua en el suelo en función del porcentaje de sodio intercambiable, este último estimado con razonable precisión a través de la relación de adsorción de sodio del extracto de saturación del suelo (Richards, 1954, Pereira et al., 1982, Rieu et al., 1991).

El presente trabajo tuvo como principal objetivo estudiar el efecto del contenido de sodio intercambiable en el suelo sobre la conductividad hidráulica del mismo.

\section{MATERIAL Y METODOS}

El efecto del porcentaje de sodio intercambiable (PSI) sobre la conductividad hidráulica de un suelo saturado, fue estudiado en condiciones de laboratorio, determinándose la relación entre la conductividad hidráulica medida en muestras de un suelo normal $\left(\mathrm{K}_{0}\right)$ y la conductividad hidráulica medida en las mismas muestras saturadas con diferentes contenidos de sodio $\left(\mathrm{K}_{\mathrm{n}}\right)$. Para esto, se llevó a cabo un experimento que constó de las siguientes etapas:

Caracterización del suelo: Se colectaron muestras de suelo no deformado utilizando anillos cilíndricos de PVC (diámetro interno de 4,7 cm y altura de 5,0 cm) introducidos en el suelo a través de un barreno tipo Uhland. en el intervalo de profundidad de $0-15 \mathrm{~cm}$, en un lisímetro de drenaje de 1,25 x 1,25 m, conteniendo en su interior un suelo aluvial de textura franco arenosa. El lisímetro estaba instalado en el "Perímetro Irrigado de São Gonçalo", Estado de la Paraíba, Brasil, y fue utilizado por Queiroz (1990) en un estudio previo. Los análisis físicos e químicos del suelo fueron realizados en el Laboratório de Iriga- ção e Salinidade del DEAg/CCT/UFCG en Campina Grande, PB. La granulometría fue realizada por el método del hidrómetro, la densidad global por el método del cilindro y la capacidad de campo y el punto de marchitez permanente a través de extractores de humedad de Richards, todos estos métodos descritos por Black (1985). Las propiedades químicas del suelo fueron determinadas de acuerdo con los procedimientos recomendados por la EMBRAPA (1997). Las características físicas y químicas del suelo son mostradas en la Tabla 1.

Tabla 1. Características físicas y químicas del suelo utilizado

\begin{tabular}{lc}
\hline Caracteristica & Valor \\
\hline Arena $\left(\mathrm{g} \mathrm{kg}^{-1}\right)$ & 608,0 \\
Limo $\left(\mathrm{g} \mathrm{kg}^{-1}\right)$ & 315,0 \\
Arcilla $\left(\mathrm{g} \mathrm{kg}^{-1}\right)$ & 77,0 \\
Densidad Global & 1,32 \\
Densidad de las Partículas & 2,60 \\
Capacidad de Campo $\left(\mathrm{g} \mathrm{kg}^{-1}\right)$ & 157,5 \\
Punto de Marcitez Permanente & 69,9 \\
$\quad\left(\mathrm{~g} \mathrm{~kg}^{-1}\right)$ & Ilita, Caulinita y \\
Composición mineralógica de la & Montmorilonita \\
fracción arcilla & 6,5 \\
pH $\left(\mathrm{H}_{2} 01: 2,5\right)$ & 12,10 \\
Capacidad de Intercambio de & 6,50 \\
$\quad$ Cationes $\left(\mathrm{cmol}_{\mathbf{c}} \mathrm{kg}^{-1}\right)$ & 2,80 \\
Calcio $\left(\mathrm{cmol}_{\mathrm{c}} \mathrm{kg}^{-1}\right)$ & 0,87 \\
Magnesio $\left(\mathrm{cmol}_{\mathrm{c}} \mathrm{kg}^{-1}\right)$ & 0,12 \\
Potasio $\left(\mathrm{cmol}_{\mathrm{c}} \mathrm{kg}^{-1}\right)$ & 0,99 \\
Sodio $\left(\mathrm{cmol}_{\mathrm{c}} \mathrm{kg}^{-1}\right)$ & \\
Porcentaje de Sodio & \\
Intercambiable $(\mathrm{PSI})$ & \\
\hline PSI $=($ Sodio intercambiable/Capacidad de intercambio de cationes) x 100
\end{tabular}

Sodificación del suelo: Para obtener diferentes PSI's el suelo fue sometido a un proceso de sodificación, el cual fue iniciado con la saturación de la muestra por capilaridad en soluciones de carbonato de sodio de diferentes concentraciones, a partir de la base de la columna, para una mejor expulsión del aire, completándose en seguida el volumen de solución en el recipiente hasta que la muestra quedara totalmente sumergida. Se salínizaron las muestras de suelo con soluciones de $\mathrm{Na}_{2} \mathrm{CO}_{3} 0,005 ; 0,015 ; 0,020 ; 0,025 ; 0,035 ; 0,050$; 0.075 e $0.100 \mathrm{M}$ para un tiempo de contacto de 8 (ocho) días. Después de la sodificación del suelo se determinó el PSI de las muestras de suelo, presentando-se estas porcentajes de sodio intercambiable entre 4,8 e 64,1 .

Determinación de la conductividad hidráulica saturada: Antes y después de la sodificación de las muestras fue determinada la conductividad hidráulica en condiciones de saturación a través del método del permeámetro de carga constante en un aparato experimental especialmente adaptado por el Laboratório de Irrigação e Salinidade (CCT/UFCG) para los objetivos de este trabajo. La Figura 1A presenta el aparato experimental utilizado y la Figura 1B un detalle de uno de los permeámetros de carga constante de $3 \mathrm{~cm}$ de agua. Los volúmenes percolados fueron medidos periódicamente durante doce horas. La conductividad hidráulica fue determinada con agua destilada, con tres repeticiones por tratamiento y calculada a través de la ecuación de Darcy de acuerdo con la siguiente expresión:

$$
\mathrm{q}=-\mathrm{K} \nabla \Psi
$$


A.

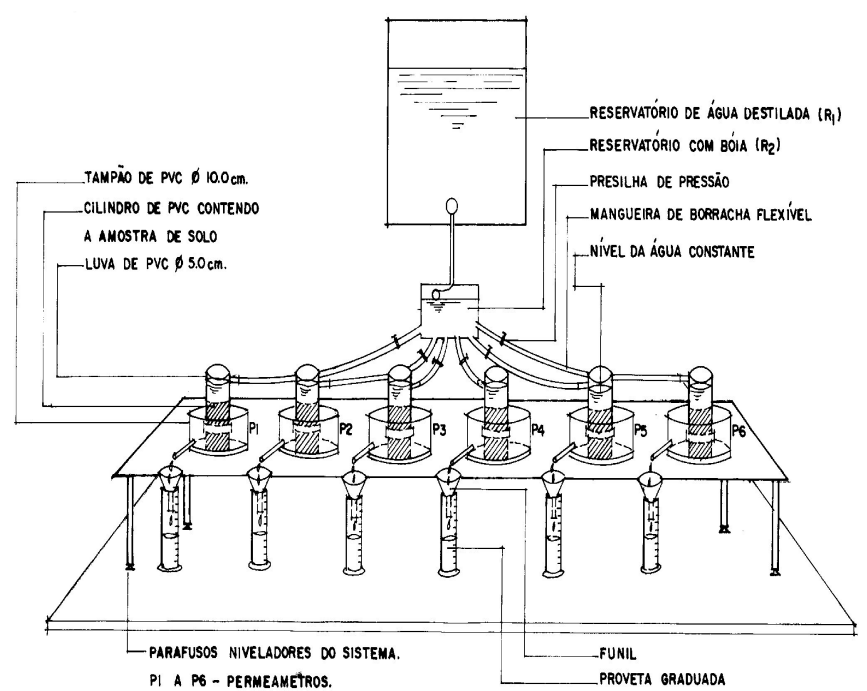

B.

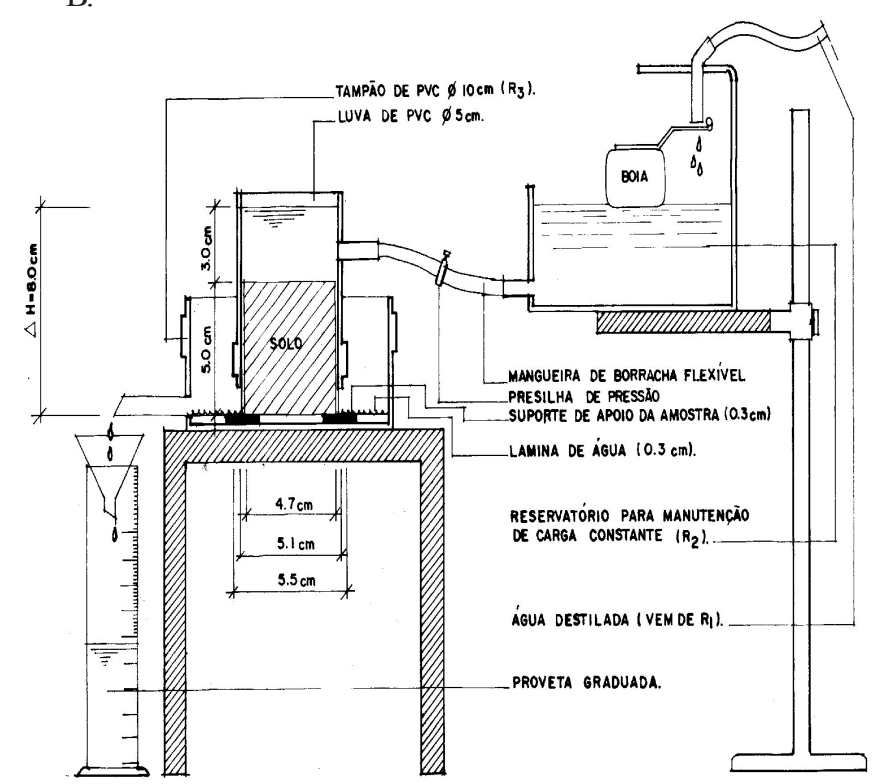

Figura 1. Batería de permeámetros utilizada para determinar la conductividad hidráulica del suelo (A) y detalle de uno de los permeámetros de carga constante (B)

donde:

$\begin{array}{ll}\mathrm{q} & \text { - Densidad de flujo, }\left(\mathrm{L} \mathrm{T}^{-1}\right) \\ \mathrm{K} & \text { - Conductividad hidráulica, }\left(\mathrm{L} \mathrm{T}^{-1}\right) \\ \nabla \Psi & \text { - Gradiente hidráulico }\end{array}$

Determinación de la conductividad hidráulica relativa (Krel): La conductividad hidráulica fue expresada como conductividad hidráulica relativa (Krel), siendo esta igual al cuociente entre esta propiedad medida al final del tratamiento $(\mathrm{Kn})$ y aquella medida al inicio de éste (Ko). Fueron efectuados análisis de variancia y de regresión entre los valores de los PSI's del suelo y los valores de Krel, siendo el grado de ajuste de los modelos evaluado a través del coeficiente de determinación $\mathrm{R}^{2}$.

\section{RESULTADOS Y DISCUSIÓN}

Las muestras de suelo saturadas con concentraciones diferentes de $\mathrm{Na}_{2} \mathrm{CO}_{3}$ durante 8 días dieron origen a suelos con porcentajes de sodio intercambiable de 4,$8 ; 9,9 ; 16,4 ; 17,4 ; 25,8$; 32,$1 ; 47,4$ e $64,1 \%$, valores considerados como tratamientos.

Las conductividades hidráulicas de las muestras de suelo medidas antes y después de la sodificación son presentadas en la Tabla 2.

Tabla 2. Valores medios* de la conductividad hidráulica inicial (Ko), final (Kn) y relativa (Krel) para los porcentajes de sodio intercambiable (PSI) estudiados

\begin{tabular}{crrrr}
\hline Tratamento & $\begin{array}{r}\text { PSI } \\
(\%)\end{array}$ & $\mathrm{K}_{0}$ & $\mathrm{~K}_{\mathrm{n}}$ & $\begin{array}{c}\mathrm{K}_{\text {rel }} \\
(\%)\end{array}$ \\
\hline T1 & 4,8 & 2,76 & 2,04 & 73,91 \\
T2 & 9,9 & 2,12 & 0,97 & 45,75 \\
T3 & 16,4 & 2,37 & 1,01 & 42,61 \\
T4 & 17,4 & 2,25 & 0,75 & 33,33 \\
T5 & 25,8 & 1,85 & 0,23 & 12,43 \\
T6 & 32,1 & 3,38 & 0,36 & 10,65 \\
T7 & 47,4 & 2,23 & 0,13 & 5,83 \\
T8 & 64,1 & 3,61 & 0,11 & 3,04 \\
\hline
\end{tabular}

"Media de tres repeticiones

${ }^{* *} \mathrm{~K}_{\mathrm{rel}}=\mathrm{K}_{\mathrm{n}} / \mathrm{K}_{0}$

Considerando que las muestras de suelo provienen de un mismo lisímetro y que para las determinaciones de la conductividad hidráulica inicial $\left(\mathrm{K}_{0}\right)$ todos los lisímetros fueron llenados de forma igual, teóricamente todos los valores deberían ser semejantes. Los altos valores de $\mathrm{K}_{0}$ encontrados en las muestras de solo referente a los tratamientos T6 y T8 se deben probablemente a variación de la compactación del suelo durante el llenado manual de los lisímetros. El coeficiente de variación para la conductividad hidráulica inicial (Ko) fue de $31,78 \%$.

Observase una visible disminución de la conductividad hidráulica final del suelo con el aumento del PSI. Esto fue verificado por McNeal \& Coleman (1966), Frenkel et al. (1978)e Park \& O‘Connor (1980). Todos reportaron una reducción de la conductividad hidráulica con el porcentaje de sodio intercambiable postulando que se debería a la expansión y/o dispersión de las arcillas con la consiguiente obstrucción de los poros conductores del suelo, sin embargo, no discuten cual es la causa más probable. Rieu et al., (1991) observó en este mismo suelo la presencia de mineral montmorilonítico, altamente expansible lo que podría justificar la primera hipótesis. La dispersión de las partículas coloidales seria producida por la baja concentración electrolítica provocada por el lavado del suelo con agua destilada durante la determinación da $\mathrm{K}_{\mathrm{n}}$. Quirk \& Schofield (1955) indican que mientras la concentración de la solución del suelo sea mantenida encima de un valor limite, la dispersión del suelo será mínima y la conductividad hidráulica será poco afectada.

La Figura 2 muestra la conductividad eléctrica (CE) del líquido percolado en cada uno de los tratamientos en función del volumen drenado.

Se observa una brusca disminución de la CE ya en el inicio del experimento, prolongándose ésta durante todo el ensayo. Así, durante el experimento los niveles de electrolitos en la solución habrían bajado del limite inferior, produciendo dispersión. Shainberg \& Letey (1984) afirman que cuando los 


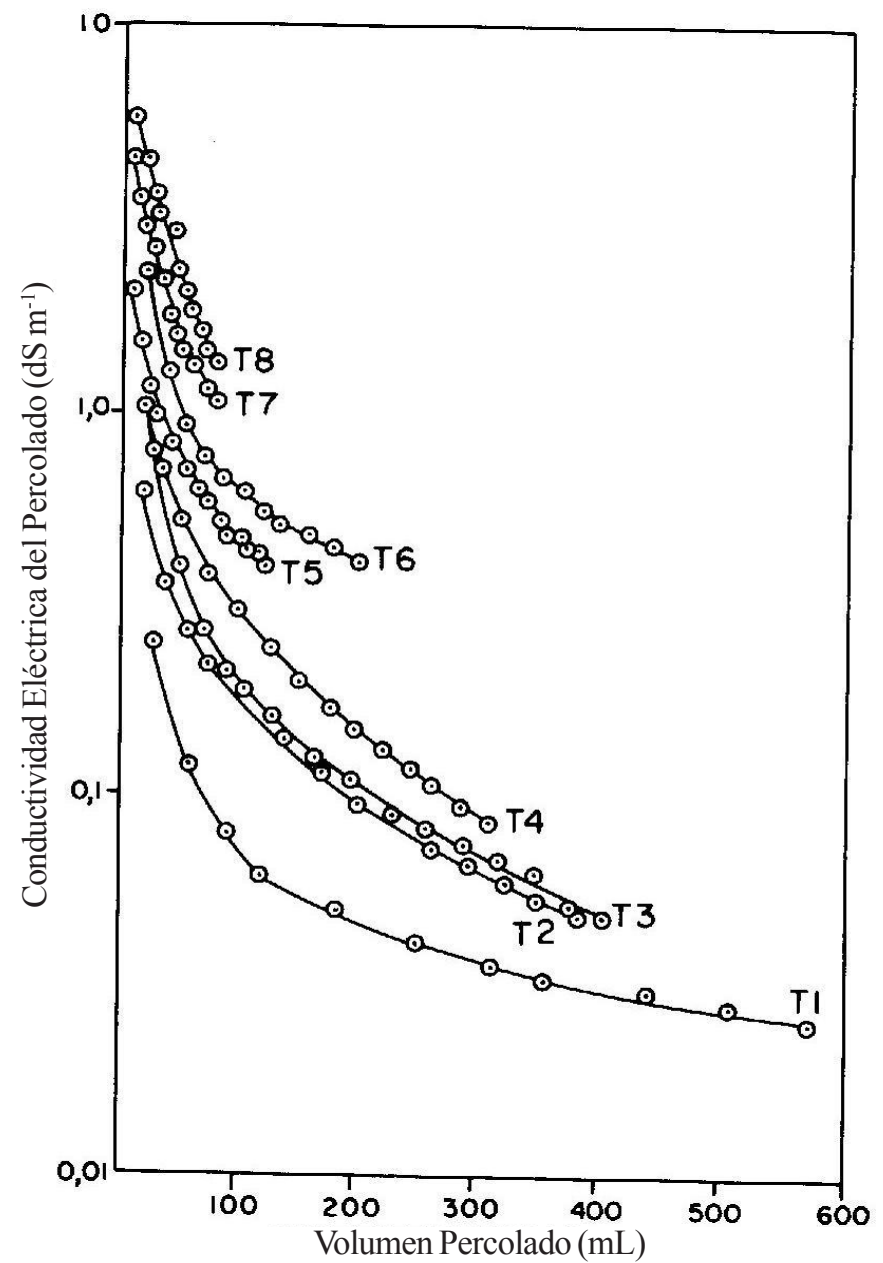

Figura 2. Conductividad eléctrica del percolado en función de su volumen, para cada tratamiento de sodificación

suelos son lixiviados con agua destilada, como fue el caso en el presente trabajo, la dispersión de las arcillas puede ocurrir con valores tan bajos de PSI cuanto 2. Para el volumen de la muestra de suelo utilizada en el presente trabajo $\left(86,85 \mathrm{~cm}^{3}\right)$, con una porosidad total de 49,00\% $\left(42,56 \mathrm{~cm}^{3}\right)$, los volúmenes drenados variaron de 0 a 14,10 volúmenes de poros.

Los resultados también mostraron una reducción de la conductividad hidráulica relativa $\left(\mathrm{K}_{\text {rel }}\right)$ con el aumento del sodio intercambiable en el suelo. La Figura 3 muestra esta relación para los ocho tratamientos. Verificase que mientras mayor el nivel de sodio intercambiable del suelo, más acentuada es la reducción de la $\mathrm{K}_{\text {rel }}$ con el volumen de agua percolado e consecuentemente con el tiempo. La muestra con un PSI de 4,8\% (T1) prácticamente no fue afectada. Aparentemente, en este caso, la concentración de sodio en el complejo de intercambio no fue lo suficientemente alta para causar la dispersión de las partículas. La variación gradual de la $\mathrm{K}_{\mathrm{rel}}$ con el aumento del PSI del suelo seria una indicación que la causa de la reducción de la conductividad hidráulica es principalmente el mecanismo de dispersión y no la expansión de las arcillas. Caso fuese esto último, este efecto se habría manifestado inmediatamente en el tratamiento $\mathrm{T} 1$ al colocar agua destilada en la muestra de suelo. Es importante considerar que en los tratamientos con bajo PSI, el volumen drenado era grande y la concentración de $\mathrm{Na}_{2} \mathrm{CO}_{3}$ utilizada para obtener el PSI deseado era menor que la del suelo, así la concentración electrolítica de la solución era baja y probablemente la dispersión del suelo prácticamente nula.

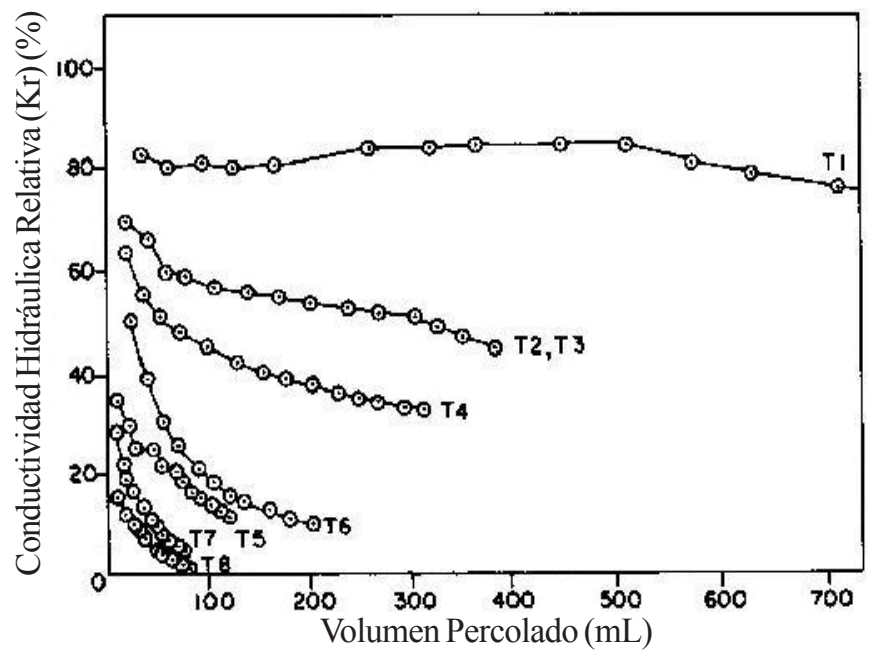

Figura 3. Relación entre la conductividad hidráulica relativa $\left(\mathrm{K}_{\mathrm{rel}}\right)$ y el volumen percolado durante el experimento, para los diferentes tratamientos

Se observa en la Figura 4 un efecto exponencial del PSI sobre la conductividad hidráulica relativa del suelo, tal cual reportado por Rieu (1983). El modelo matemático que más se ajustó a los resultados fue $\mathrm{K}_{\mathrm{r}}=88,30 \mathrm{e}^{(-0.06 \mathrm{PSI})}$ con un coeficiente de determinación $\left(\mathrm{R}^{2}\right)$ de 0,9637 , estadísticamente significativo al $0,01 \%$ de probabilidad. La Figura 4 muestra que, efectivamente, el valor de PSI igual a $15 \%$ es crítico. Cuando el PSI del suelo aumenta de 4,8 para $15 \%$ se observa que la conductividad hidráulica relativa del suelo disminuye de 73 para $38 \%$, una disminución de aproximadamente $48 \%$. Precisamente es este valor de PSI el utilizado como valor estándar para clasificar los suelos sódicos. Esto muestra la importancia de la aplicación de correctivos orgánicos y/o químicos para mantener el PSI del suelo abajo de $15 \%$ y mejorar la capacidad de infiltración de agua de los suelos.

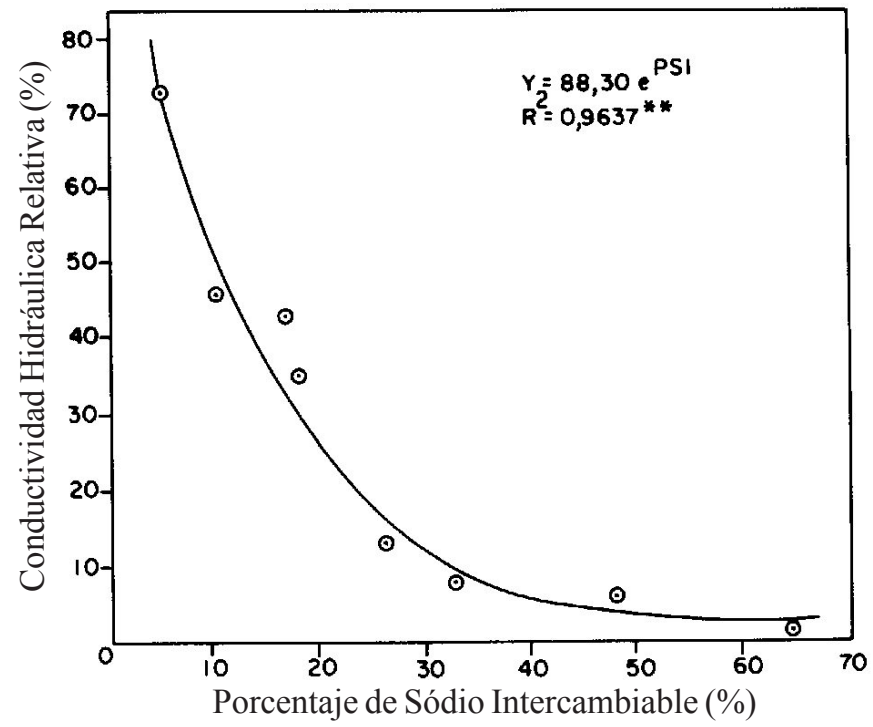

Figura 4. Relación entre la conductividad hidráulica relativa y el Porcentaje de Sodio Intercambiable (PSI) de suelo 
El analice de variancia (Teste F) reveló diferencia estadísticamente significativa al $0,01 \%$ de probabilidad para los tratamientos estudiados. Comparando las medias de $\mathrm{K}_{\mathrm{r}}$ al $0,01 \%$ de probabilidad fue verificado que la $\mathrm{K}_{\text {rel }}$ obtenida en el tratamiento $\mathrm{T} 1$ fue estadísticamente mayor que aquellas obtenidas en los otros. Los tratamientos T2, T3, y T4, no fueron diferentes entre si pero se diferenciaron de los T5, T6, T7 y T8 siendo éstos últimos iguales entre si.

\section{CONCLUSIONES}

1. La facilidad con que el agua se mueve en el suelo disminuyó con el aumento del sodio intercambiable de la solución del suelo.

2. Se obtuvo un modelo matemático que permite determinar la conductividad hidráulica del suelo a partir del conocimiento del porcentaje de sodio intercambiable de este.

\section{LITERATURA CITADA}

Bar-On, P.; Shainberg, I.; Michaeli, I. The electrophoretic mobility of $\mathrm{Na} / \mathrm{Ca}$ montmorillonite particles . Journal of Colloid and Interface Science, v.33, p.471-472. 1970.

Black, C.A. Methods of soil analysis. Part 1. Physical and mineralogical properties, including statistics of measurements and sampling. Madison: American Society of Agronomy. 1985.770p.

Chhabra, R. Soil salinity and water quality. Rotterdam, A.A. Balkema Publishers, 1996. 283p.

EMBRAPA. Empresa Brasileira de Pesquisa Agropecuária.Manual de métodos de analises de solo. 2 ed. Rio de Janeiro; Serviço Nacional de Levantamento e Conservação de Solos. 1997. 212p.

Frenkel, H; Goertzen, J.O.; Rhoades, J.O. Effects of clay type and content, exchangeable sodium percentage and electrolyte concentration on clay dispersion and soil hydraulic conductivity. Soil Science Society American Journal, Madison, v.42, p.32-39. 1978.

Gupta, R.K.; Abrol I.P. Salt affected soils: Their reclamation and management for crop production. Advances of Soil Science. v.11, p.223-228. 1990.

McNeal, B.L.; Coleman, N.T. Effect of solution composition on soil hydraulic conductivity. Soil Science Society America Proceedings, Madison, v.30, p.308-312, 1966.

Northcote, K.H.; Skene, J.K.M. Australian soils with saline and sodic properties. CSIRO, Mellbourne: CSIRO, 1972. 61p. Publication No 27
Park, C.S.; O‘Connor, G. Salinity effects on hydraulic properties of soils. Soil Science Society America Journal, Madison, v.130, p.167-174, 1980.

Pereira, F.A.M.; Medina, B.F., Gheyi, H.R. Solos afetados por sais no Nordeste. II: Correlação entre sódio solúvel e intercambiável. Revista Brasileira de Ciência do Solo, Campinas, v.6, p.167-170, 1982.

Pizarro, F. Drenaje agrícola Y recuperación de suelos salinos. Madrid: Agrícola Española, 1978, 521p.

Pupisky, H.; Shainberg, I. Salt effects on the hydraulic conductivity of a sandy soil. Soil Science Society American Journal. Madison, v.43, p.429-433, 1979.

Queiroz, M.M. Estudo da condutividade hidráulica de um solo aluvial franco-arenoso em experimentos de lisímetros. Campina Grande: UFPB, 1990. 138p. Dissertação Mestrado

Quirk, J.P.; Schofield, R.K. The effect of electrolyte concentration on soil permeability. Journal of Soil Science, Oxford, v.6, p.163-178. 1955.

Quirk, J.P. Particle interaction and soil swelling. Israeli Journal of Chemistry, Jerusalem, v.6, p.213-234, 1968.

Rengasamy, P.; Sumner, M.E. Processes involved in sodic behavior. In: Sumner, M.E.; Naidu, R. (ed) Sodic soils. New York: Oxford University Press, New York, 1997.

Rhoades, J, D. Overview: Diagnosis of salinity problems and selection of control practices. In: Tanji, K.K.(ed). Agricultural salinity assessment and management. New York: American Society of Civil Engineers. 1990. p.18-41.

Richards, L.A. (ed) Diagnosis and improvement of saline and alkali soils. Washington D.C; US Department of Agriculture, 1954. 160p. Agricultural Handbook, 60

Rieu, M. Simulation numerique des flux hydriques et prediction de la salinité dans les soils. Paris: ORSTOM, 1983. 161p.

Rieu, M.; Touma, J.; Gheyi, H.R. Sodium - calcium exchange on Brazilian soils: Modeling the variation of selectivity coefficients. Soil Science Society America. Journal, Madison, v.55,p.1294-1300, 1991.

Shainberg, I. Soil response to saline and sodic conditions. In: Tanji, K.K.(ed). Agricultural salinity assessment and management. New York: American Society of Civil Engineers. 1990. p.91-112.

Shainberg, I.; Letey, J. Response of soils to sodic and saline conditions. Hilgardia, v.52, p.1-57, 1984.

Sumner, M.E. Sodic soils; New perspectives. In: Naidu, R; Sumner, M.E.; Rengasamy, P. (eds). Australian sodic soils. Distribution, properties and management. Adelaide: First National Conference and Workshop on Sodic Soils, 1995, p.1-34. 